\title{
Solitär fibröser Tumor der Pleura assoziiert mit Doege-Potter-Syndrom
}

\author{
Pleura Solitary Fibrous Tumor Associated with Doege-Potter Syndrome
}

Autoren

Institut
M. Valenzuela Membrives, L. Cassini Gómez de Cádiz, A. Cueto Ladròn de Guevara, M. García Pacheco

San Cecilio Klinikum, Sektion Pneumologie, Granada, Spanien eingereicht 3.3 .2013 akzeptiert nach Revision 15.4.2013

Bibliografie

DOI http://dx.doi.org/

10.1055/s-0033-1344079

Pneumologie 2013; 67: 340-343

(c) Georg Thieme Verlag KG Stuttgart · New York ISSN 0934-8387

Korrespondenzadresse Dr. med. Ma del Mar Valenzuela Membrives Hospital Universitario San Cecilio, Neumología Calle Doctor Olóriz 16 18012 Granada Spanien

marivalen8@hotmail.com

\section{Zusammenfassung \\ $\nabla$}

Unter einem Doege-Potter-Syndrom wird die Kombination aus einem solitären fibrösen Tumor und symptomatischen Hypoglykämien verstanden. Der solitäre fibrinöse Tumor ist eine mesenchymale Neoplasie, welche primär der Pleura zuzuordnen ist, prinzipiell aber in jedem Organ oder Gewebe mit CD34+-Stromazellen vorkommen kann. Die meisten Patienten sind zum Zeitpunkt der Diagnose asymptomatisch. Es wird von klinischen Beschwerden wie Husten, Dyspnoe, Brustschmerzen und Hypoglykämien aufgrund einer Produktion von IGF-2 berichtet (Doege-PotterSyndrom). Andere paraneoplastische Erscheinungen wie Osteoarthropathie und Galaktorrhö sind ebenfalls möglich. Wir berichten über eine 77jährige Patientin mit einem solitär fibrösen Tumor der Pleura sowie symptomatischen Hypoglykämien. Als primäre Behandlung sollte immer eine chirurgische Resektion erfolgen. Langjährige Kontrolluntersuchungen sind notwendig, da Rezidive auch nach langer Zeit der Remission auftreten können.

\section{Einleitung}

Der solitäre fibröse Tumor (SFT) stellt eine sehr seltene Form einer mesenchymalen Neoplasie dar. Die Tumore können, auch ohne Charakteristika eines malignen Tumors, rasch wachsen und entsprechend ihrer Lokalisation klinische Symptome verursachen (Husten, Dyspnoe, Schmerzen). Gelegentlich leiden die Patienten zusätzlich unter Hypoglykämien, da der Tumor den Insulin-like growth Faktor 2 (ILGF-2) sezernieren kann.

\section{Abstract \\ $\nabla$}

The Doege-Potter Syndrome is known as a combination of intrathoracic tumor associated to symptomatic hypoglycemia. Solitary Fibrous Tumor is a mesenchymal neoplasm. Initially, it was described in the pleura, however, it starts out in any organ or tissue at the expense of a stromal CD34+ cell. Most of the patients are asymptomatic at the time of diagnosis. Symptoms such as cough, dyspnea, chest pain, and hypoglycemia due to production of IGF-2 have been reported ( DoeggePotter Syndrome). Others described paraneoplastic profiles are hypertrophic osteoarthropathy and in some cases galactorrhea. We report on a case of a 77 year old patient with solitary fibrous tumor in the pleura as well as symptomatic hypoglycemia. The primary treatment is surgical resection, and a longtime monitoring is necessary because recurrences can occur even after a long period of remission.

\section{Fallbericht}

Wir berichten über eine 77-jährige Patientin, Nichtraucherin und Hausfrau, ohne direkten Kontakt zu definierten Noxen (Asbest). Anamnestisch bestanden ein Bluthochdruck, eine Arthrose, eine chronische Veneninsuffizienz, eine Retinopathie und ein Zustand nach einem Endometriumkarzinom, welches 1996 mit Chemo- und Radiotherapie behandelt worden war.

Die Patientin klagte im November 2011 erstmals über Belastungsdyspnoe.

Im Verlauf der folgenden 6 Monaten entwickelte die Patientin eine ausgeprägte Inappetenz und einen Gewichtsverlust von $10 \mathrm{~kg}$. 

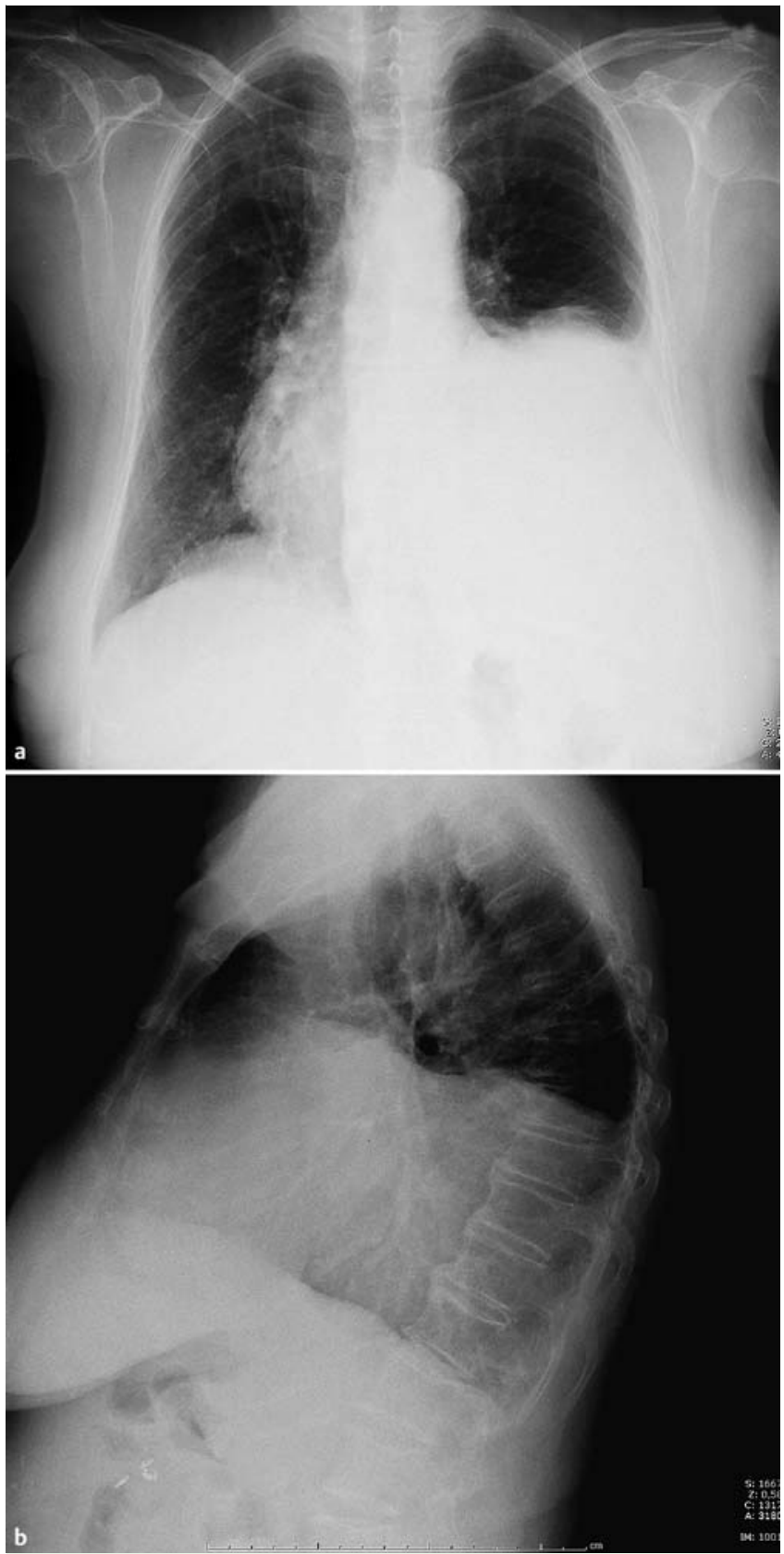

Abb. 1 Röntgen des Thorax mit Transparenzminderung über 2/3 des linksseitigen Hemithorax.

Im Mai 2012 wurde die Patientin mit akuter Dyspnoe in Verbindung mit linksthorakalen Schmerzen in unsere Notaufnahme eingeliefert.

Beim Aufnahmegespräch berichtete die Patientin, seit April 2012 rezidivierend an Schwäche, Schwitzen und einem eingeschränkten Sehvermögen zu leiden. Bei einer Konsultation beim Hausarzt sei bei einem solchen Ereignis ein Blutzucker von $50 \mathrm{mg} / \mathrm{d}$ gemessen worden und eine Glucoseinfusion gegeben worden.

Zum Zeitpunkt der Aufnahme zeigte sich laborchemisch ein Blutzuckerspiegel von $58 \mathrm{mg} / \mathrm{dl}$ und ein Kaliumgehalt von 2,6 mmol/L, alle anderen Werte, inklusive der kardialen Marker, befanden sich im Normbereich. Im Rahmen der weiterführenden Untersuchungen imponierte eine deutliche Monozytose bei normalen Leukozyten. Weiterhin zeigte sich eine deutliche Erhöhung des Tumormarkers CA 125 auf $41,37 \mathrm{U} / \mathrm{ml}$.

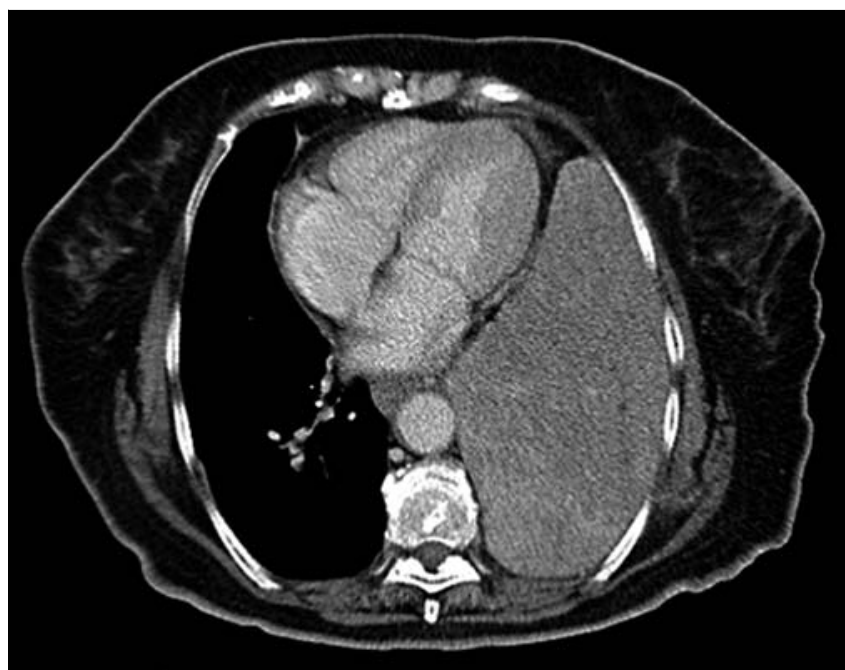

Abb.2 Computertomografie des Thorax mit ausgedehnter Tumormasse im linken Hemithorax.

Die Röntgen-Thorax-Aufnahme zeigte eine ausgedehnte Infiltration im linken Mittel- und Untergeschoss ( $\bullet$ Abb. 1 a und $\mathbf{b})$. Sonografisch konnte kein Pleuraerguss nachgewiesen werden. In der Computertomografie stellte sich ein großer inhomogener Tumor dar $(13,5 \times 17,5 \times 9,5 \mathrm{~cm})(\bullet$ Abb. 2$)$.

Bei der Lungenfunktionsprüfung zeigt sich eine moderate restriktive Ventilationsstörung mit einer peripheren, nicht reversiblen Obstruktion. In der Blutgasanalyse imponierte eine leichte Hypoxämie mit Normokapnie.

Durch eine CT-gestützte perkutane Biopsie konnte ein fibröser Tumor mit dem folgenden immunhistochemischen Profil festgestellt werden: positiv für CD34, CD99, BCL2 und Vimentin und negativ für S100, CD117, CD10, Inhibina, WT1 und Desmin. Die Wachstumfraktion (KI67) lag unter $1 \%$.

Der Fall wurde den Kollegen der Thoraxchirurgie vorgestellt, und es wurde eine primäre Resektion beschlossen. Intraoperativ fand sich eine Tumormasse mit einem Durchmesser von $20 \mathrm{~cm}$ mit einer fraglichen Infiltration der Pleura, des Zwerchfells und des Perikards. Es erfolgte eine fraktionierte Tumorresektion.

Das erste Resektat war von weiß-brauner Farbe mit einer Größe von $15 \times 15 \times 8 \mathrm{~cm}$. Das zweite Resektat war $10 \times 7 \times 6,5 \mathrm{~cm}$ groß, mit einem zusätzlichen muskulösen Fragment an der Basis von $4 \times 3 \mathrm{~cm}$. Das dritte Präparat zeigte eine Infiltration des Perikards. Die mikroskopische Diagnose von allen Resektaten bestätigte das Vorliegen eines solitären fibrösen Tumors, nun allerdings mit einer Wachstumfraktion (KI67) von 10\% ( $\bullet$ Abb.3).

Einen Monat nach der chirurgischen Therapie war die Patientin frei von Symptomen. Hypoglykämien traten nicht mehr auf. Das postoperative Röntgenbild zeigte nach einem Monat keine Hinweise auf ein Rezidiv ( $\bullet$ Abb.4).

\section{Diskussion \\ $\nabla$}

Der solitäre fibröse Tumor (SFT) wurde im Jahr 1931 von Klemperer und Rabin [1] als lokale und gutartige Läsion beschrieben. Weitere Studien unter der Leitung von Murray [2] deuteten auf einen Ursprung aus Mesothelzellen hin. Später wurde bestätigt, dass in den Tumorzellen epitheliale Marker (Zytokeratine und epitheliale Membranantigene) fehlen. Hingegen zeigten sich fibroblastische Stromazell-Marker, wie Vimentin und CD34+. 


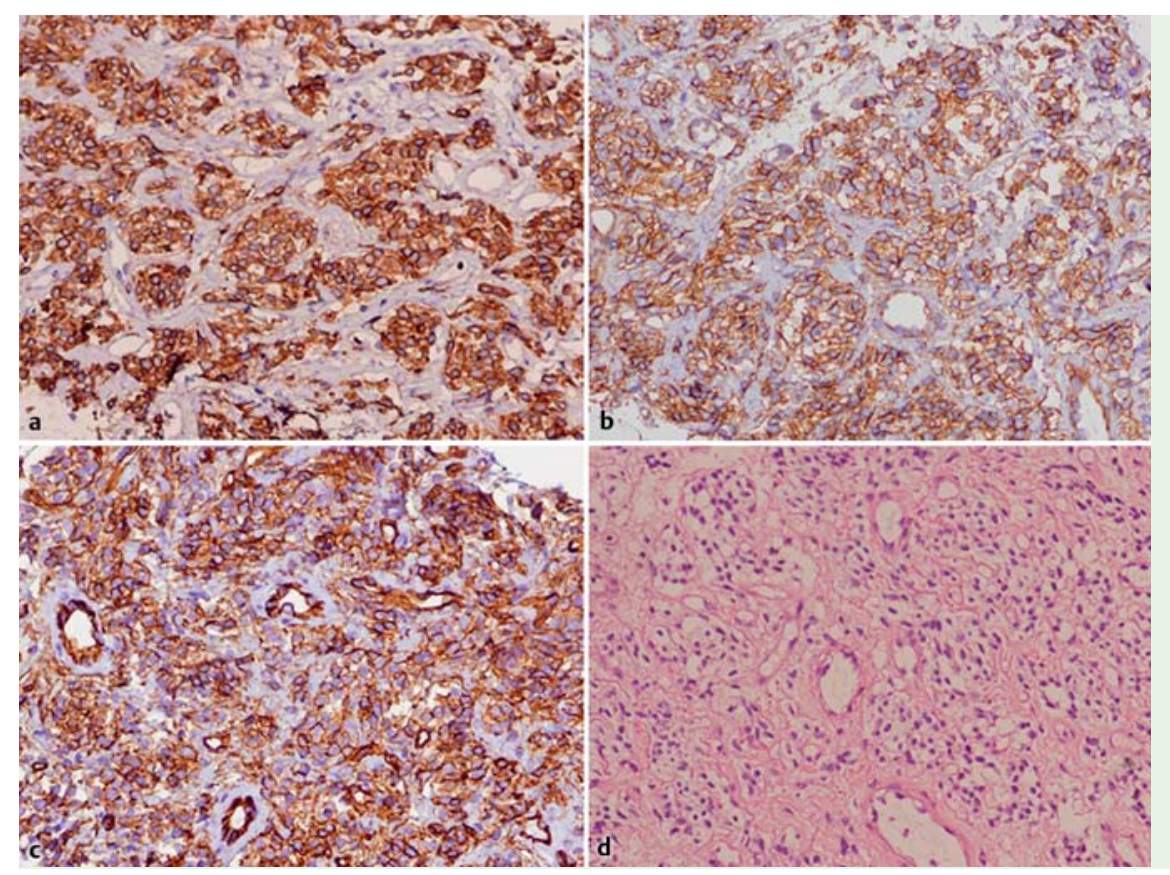

Abb.3 Das immunhistochemische Profil des fibrösen solitären Tumors.

a Bcl2, b CD34, c CD99, d Haematoxylin-Eosin.

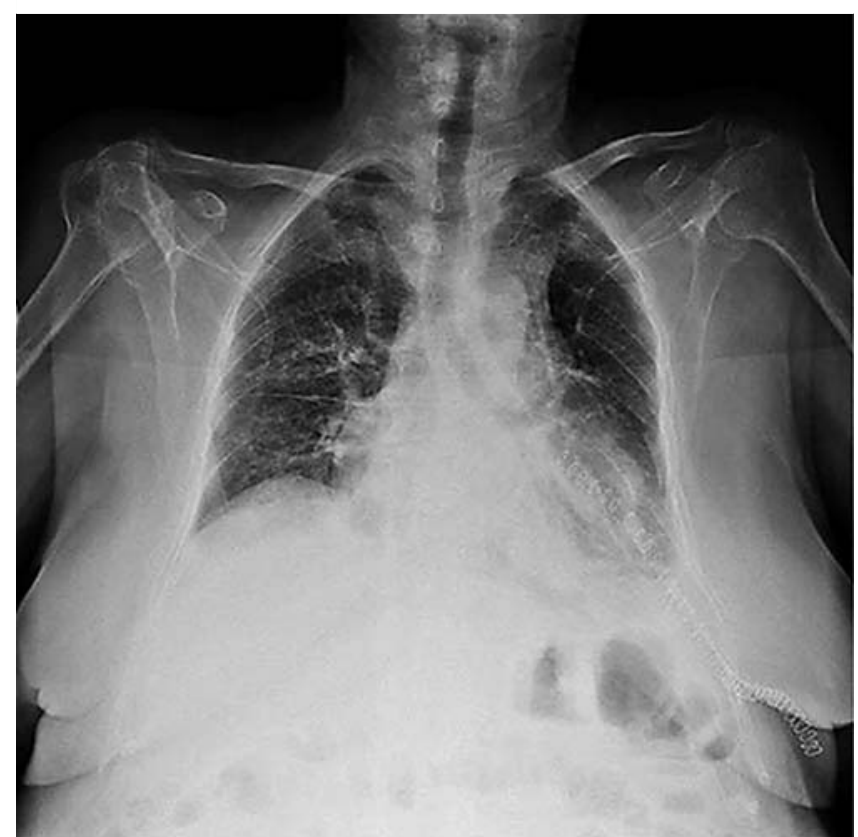

Abb.4 Postoperative Röntgenaufnahme des Thorax ohne Hinweis auf Rezidiv oder Komplikationen.

Es wird angenommen, dass die histologische Herkunft in einer allgegenwärtigen, interstitiellen, myofibroblastischen CD34+Zelle liegt [3]. Dies würde erklären, warum der Tumor an verschiedenen Orten beschrieben wurde: Augenhöhle, Schilddrüse, Leber, Pankreas, Retroperitoneum, Haut, Nieren, Periost, Knochen usw. Die Tumoren erscheinen als eine solide, sessile, gestielte, gräulich-weiße Läsion, ohne Invasion in benachbarte Organe, mit oder ohne Nekrosen und Blutungen. Die histologischen Bilder können sehr unterschiedlich sein, wobei die Unterscheidung zwischen benignen und malignen Formen schwierig sein kann. Ein SFT, der gleich oder größer als $10 \mathrm{~cm}$ ist, benachbarte Strukturen infiltriert und mehr als vier anormale Mitosen pro Gesichtsfeld zeigt, gilt als malignom-verdächtig. Weitere Zeichen potenzieller Malignität können die folgenden Merkmale sein: Hyperzellularität, Pleomorphismus, epitheloide sarkomatöse Muster, vaskuläre Invasion und Bereiche mit Einblutungen oder Nekrosen [4].

Bei benignen SFT finden sich hingegen häufig Mesenchymzellen, kombiniert mit Kollagenfasern vom Typ des Keloid und Pedikel, die gut abgrenzbar sind. Ein nukleärer Pleomorphismus und mitotische Aktivitäten fehlen.

Der SFT hat keine spezifischen Marker. In der Regel sind die Zellen positiv auf Vimentin und CD34+, die allerdings auch in Dermatofibrom, Dermatofibrosarkom und Hämangioperizytom darstellbar sind. BCL-2, CD99 und Vimentin sind in den meisten Fällen positiv, und sie sind sehr hilfreich, wenn die folgenden Antigene negativ sind: S-100-Protein, Desmin, Muskel-spezifisches Antigen, Cytokeratin und CD68 [6].

Wenn ein hämangioperizytoides Muster überwiegt, sollte eine Differenzialdiagnose zwischen benignen und malignen Läsionen wie dem Angiofibrom und dem mesenchymalen Condrosarkom durchgeführt werden. Gengler und andere Autoren glauben, dass das SFT und das Hämangioperizytom Varianten derselben Läsion sind [7].

Im Jahre 1930 beschrieben zwei amerikanische Ärzte, Karl Doege und Roy Potter, das gemeinsame Auftreten von einem mediastinalen fibrösen Tumor und Hypoglykämie bei einem Patienten [8]. Das sogenannte Doege-Potter-Syndrom (DPS) ist sehr selten und in den meisten Fällen mit intrathorakalen fibrösen Tumoren assoziiert, allerdings sind auch Fälle mit mesenchymalen Tumoren beschrieben worden [9]. Der SFT kann den Insulin-like GrowthFactor-1 und -2 (IGF-1, IGF2) sezernieren, die in der Folge durch eine Aktivierung des Insulinrezeptors und eine Rezeptor-Hemmung von Glykogen in der Leber Hypoglykämien provozieren können $[1,10]$. Es sind Fälle von SFT beschrieben, bei denen eine Insulinresistenz letztlich zu letalen Verläufen geführt hat [11,12]. Der SFT wird auch mit der hypertrophen Osteoarthropathie Pierre Marie in Verbindung gebracht [13].

Im Röntgen-Thorax-Bild stellen sich SFT meist als solitäre Knoten, klar abgegrenzt und gelappt in der Lungenperipherie oder in den Lappenspalten dar. Ein tumorassoziierter Zwerchfellhoch- 
stand kann radiologisch ebenfalls nachweisbar sein. Im CT wird der SFT als eine nicht-invasive, homogene, weiche Gewebemasse beschrieben. Kleinere Läsionen bilden stumpfe Winkel mit der Pleura. Im Gegensatz hierzu sind größere Läsionen häufig heterogen und bilden spitze Winkel mit der benachbarten Pleura. Es fehlen definierte radiologische Kriterien zur Unterscheidung von malignen und benignen Läsionen, auch wenn heterogene Läsionen, Tumoren mit Mediastinalverlagerung und begleitende Pleuraergüsse häufiger bei malignen Läsionen zu finden sind [10]. Die Therapie der Wahl ist immer die chirurgische Resektion des Tumors. Die Tumorresektion stellt in der Regel einen kurativen Ansatz dar und führt auch zum Verschwinden der klinischen Symptome (Hypoglykämien). In der Regel können die SFT atypisch reseziert werden. Nur in einzelnen Fällen ist eine Segmentoder Lappen-Resektion notwendig [15]. Rezidive kleiner SFT sind selten (2\%) [16]. SFT von mehr als $10 \mathrm{~cm}$ und mit hoher mitotischer Aktivität haben allerdings Rezidivraten von bis zu $80 \%$ und müssen engmaschig postoperativ nachbeobachtet werden.

\section{Interessenkonflikt}

$\nabla$

M. Valenzuela, L. Cassini, A. Cueto und M. García geben an, dass kein Interessenkonflikt besteht.

\section{Literatur}

1 Klemperer P, Rabin CB. Primary neoplasms of the pleura: a report of five cases. Arch Pathol 1931; 11: 385-412

2 Stout AP, Murray MR. Localized mesothelioma. Arch Pathol 1942; 34: 951-964

3 McClure J. Advances in the understanding of the molecular pathology of connective tissue neoplasms. J Pathol 1997; 181: 357-358
4 England DM, Hochholzer L, McCarthy MJ. Localized benign and malignant fibrous tumours of the pleura: a clinicopathologic review of 223 cases. Am J Surg Pathol 1989; 13: 640-658

5 Gutiérrez-Diaz Ceballos ME, Hernández Solís A, Cruz-Ortiz H. Tumor fibroso solitario. Estudio clinico-patológico de 16 casos. Cir Cir 2011; 79: $417-423$

6 Jorge DL, Ramos-Salazar P, Millán-Ruiz V et al. Tumor fibroso solitario. Estudio histopatológico e inmunohistoquímico de 18 casos de localización extrapleural. Patol Rev latinoam 2010; 48: 73-81

7 Gengler C, Guillou L. Solitary fibrous tumour and haemangiopericytoma; evolution of a concept. Histopathology 2006; 48: 63-74

8 Doege KW. Fibrosarcoma of the mediastinum. Ann Surg 1930; 92 : 955-960

9 Milenkovic BA, Stojsic JM, Motohiko A et al. The role of IGF II in Doege Potter Syndrome: A propos of two cases. Chest 2008; 134: c17002

10 Chang ED, Lee EH, Won YS et al. Malignant solitary fibrous tumor of the pleura causing recurrent hypoglycemia: immunohistochemical stain of insulin like growth factor I receptor in three cases. J Korean Med Sci 2001; 16: 220-224

11 Zafar H, Takimoto CH, Weiss G. Doege-Potter syndrome: hypoglycemia associated with malignant solitary fibrous tumor. Med Oncol 2003; 20: 403-408

12 Kishi K, Homma S, Tanimura S. Hypoglycemia induced by secretion of high molecular weight insulin-like growth factor II from a malignant solitary fibrous tumor of the pleura. Intern med 2001; 40: 41-44

13 Fridlington J, Weaver J, Kelly $B$ et al. Secondary hypertrophic osteoarthropathy associated with solitary fibrous tumor of the lung. J Am Acad Dermatol 2007; 57: 5106-5110

14 Nagasako Y, Misawa K, Kohashi S et al. Solitary fibrous tumors in the retroperitoneun. J Am Coll Surg 2004; 198: 322 - 323

15 Golf JS, Antonescu CR, Hadju C et al. Clinico pathologic correlates of solitary fibrous tumors. Cancer 2002; 94: 1057-1068

16 Julve Parreño A, Ponce Pérez E, Dosdá Muñoz R. Tumor fibroso localizado de da pelura: hallazgos radiológicos. Radiología 2007; 49: 339-342 